\title{
LHC Collimation: Design and Results from Prototyping and Beam Tests
}

\author{
O. Aberle, G. Arduini, R. Assmann, I. Baishev ${ }^{1)}$, A. Bertarelli, H. Braun, M. Brugger, \\ H. Burkhardt, S. Calatroni, F. Caspers, E. Chiaveri, A. Dallochio, B. Dehning, A. Ferrari, \\ M. Gasior, A. Grudiev, E.B. Holzer, J.B. Jeanneret, J.M. Jimenez, Y. Kadi, I. Kurochkin ${ }^{1)}$, \\ R. Losito, M. Magistris, A. Masi, M. Mayer, E. Metral, R. Perret, C. Rathjen, S. Redaelli, \\ G. Robert-Demolaize, S. Roesler, M. Santana, D. Schulte, G. Spiezia" ${ }^{2)}$, P. Sievers, K. Tsoulou, \\ H. Vincke, V. Vlachoudis, J. Wenninger \\ CERN, Geneva, Switzerland
}

\begin{abstract}
The problem of collimation and beam cleaning is one of the most challenging aspects of the LHC project. A collimation system must be designed, built, installed and commissioned with parameters that extend the present state-of-the-art by 2-3 orders of magnitude. Problems include robustness, cleaning efficiency, impedance and operational aspects. A strong design effort has been performed at CERN over the last two years. The adopted phased approach is described. Robust and precisely controllable collimators have been designed. Several LHC prototype collimators have been built and tested with the highest beam intensities that are presently available at CERN. The successful beam tests are presented, including beam-based setup procedures, a 2 MJ robustness test and measurements of the collimator-induced impedance. Finally, an outlook is presented on the challenges that are ahead in the coming years.
\end{abstract}

${ }^{1)}$ IHEP, Protvino, Russia

${ }^{2)}$ Naples University Federico II, Naples, Italy

Presented at PAC 2005, Knoxville, USA, May 16-20, 2005

CERN,

CH-1211 Geneva 23,

Switzerland

Geneva, June 2005 


\title{
LHC COLLIMATION: DESIGN AND RESULTS FROM PROTOTYPING AND BEAM TESTS
}

\author{
R. Assmann, O. Aberle, G. Arduini, A. Bertarelli, H. Braun, M. Brugger, H. Burkhardt, S. Calatroni, \\ F. Caspers, E. Chiaveri, A. Dallochio, B. Dehning, A. Ferrari, M. Gasior, A. Grudiev, E. B. Holzer, \\ J.B. Jeanneret, J.M. Jimenez, Y. Kadi, R. Losito, M. Magistris, A. Masi, M. Mayer, E. Métral, \\ R. Perret, C. Rathjen, S. Redaelli, G. Robert-Demolaize, S. Roesler, M. Santana, D. Schulte, \\ P. Sievers, K. Tsoulou, H. Vincke, V. Vlachoudis, J. Wenninger, CERN, Geneva, Switzerland \\ I. Baishev, I. Kurochkin, IHEP, Protvino, Russia \\ G. Spiezia, Naples University Federico II, Napoli.
}

\begin{abstract}
The problem of collimation and beam cleaning is one of the most challenging aspects of the LHC project. A collimation system must be designed, built, installed and commissioned with parameters that extend the present state-of-the-art by 2-3 orders of magnitude. Problems include robustness, cleaning efficiency, impedance and operational aspects. A strong design effort has been performed at CERN over the last two years. The adopted phased approach is described. Robust and precisely controllable collimators have been designed. Several LHC prototype collimators have been built and tested with the highest beam intensities that are presently available at CERN. The successful beam tests are presented, including beam-based setup procedures, a 2 MJ robustness test and measurements of the collimator-induced impedance. Finally, an outlook is presented on the challenges that are ahead in the coming years.
\end{abstract}

\section{INTRODUCTION}

The LHC requires a stored energy of $360 \mathrm{MJ}$ per beam. This is 2-3 orders of magnitudes above the stored energy that is handled in other proton colliders and must be compared to a typical $10 \mathrm{~mJ} / \mathrm{cm}^{3}$ quench limit of the superconducting magnets. A robust and highly efficient collimation system is required to withstand the high beam intensities and to absorb unavoidable beam losses [1]. At $7 \mathrm{TeV}$ the specified peak loss rate corresponds to a total loss in beam intensity of $1 \%$ over $10 \mathrm{~s}$. This loss rate implies cleaning inefficiencies of around $5 \times 10^{-4}$. Proton losses must be intercepted at the collimators and not more than $0.05 \%$ of the impacting protons are allowed to leak out of the "cleaning insertions". Two such cleaning insertions have been included into the LHC layout for betatron and momentum cleaning [2].

\section{LHC COLLIMATION DESIGN}

The LHC collimation design has been revisited. Important improvements have been implemented. A complete system description would go beyond the scope of this paper. However, we summarize the key features:

1. Robust primary and secondary collimators with $1.2 \mathrm{~m}$ long water-cooled carbon-carbon (CC) jaws (this includes $0.2 \mathrm{~m}$ of tapering), precise jaw position control and handling of image currents [3]. These col- limators intercept the primary and secondary beam halos and are the closest aperture to the circulating beam.

2. Movable absorbers ("high-Z collimators") with $1.2 \mathrm{~m}$ long water-cooled, tapered $\mathrm{Cu} / \mathrm{W}$ jaws. Absorbers intercept the tertiary beam halo and/or the shower products from the cleaning insertions or the p-p collisions. Absorbers have been integrated downstream of the cleaning insertions and in all experimental insertions.

In each of the two LHC rings 46 collimators and absorbers will be installed. The 92 collimators in total have been carefully positioned to respect space constraints while providing optimal cleaning efficiency and good machine protection.

The usage of the non-metallic CC collimators close to the LHC beam (full gaps down to $3 \mathrm{~mm}$ ) optimizes robustness for the price of high induced impedance. Though this collimator-induced impedance was almost halved by proper positioning of the collimators, the overall effect is still strong and is estimated to limit the LHC proton beam intensities at around $40 \%$ of their nominal values [4].

The robust but impedance-limited initial LHC collimation system is called "phase 1". In order to overcome the limitations in LHC performance the phase 1 secondary collimators are complemented by space reservations for $1.48 \mathrm{~m}$ long "phase 2" collimator tanks. In total 30 space reservations will allow to complement the collimation system in 2010 with advanced "hybrid" collimators that combine sufficient reliability with low impedance. The R\&D on suitable phase 2 collimators is already on its way at CERN and in the US (LARP program).

Other important design work on the collimation system concerns energy depositions studies [5] and analysis of associated radiation issues [6]. A first result of cleaning efficiency with the full system is included in [7].

\section{PROTOTYPING}

The design of the robust CC collimators for the LHC is described in [3]. Two prototypes were built for installation into the SPS ring and the TI8 injection line at CERN. Photographs of the fully equipped LHC collimator are shown in Figures 1 and 2.

The collimator design team had to address challenging requirements including jaw flatness $(25 \mu \mathrm{m}$ over $1 \mathrm{~m})$, heat conductance $\left(\sim 10 \mathrm{~kW} / \mathrm{m}^{2} / \mathrm{K}\right)$, mechanical plays $(\sim 10 \mu \mathrm{m})$ and clean vacuum performance. 


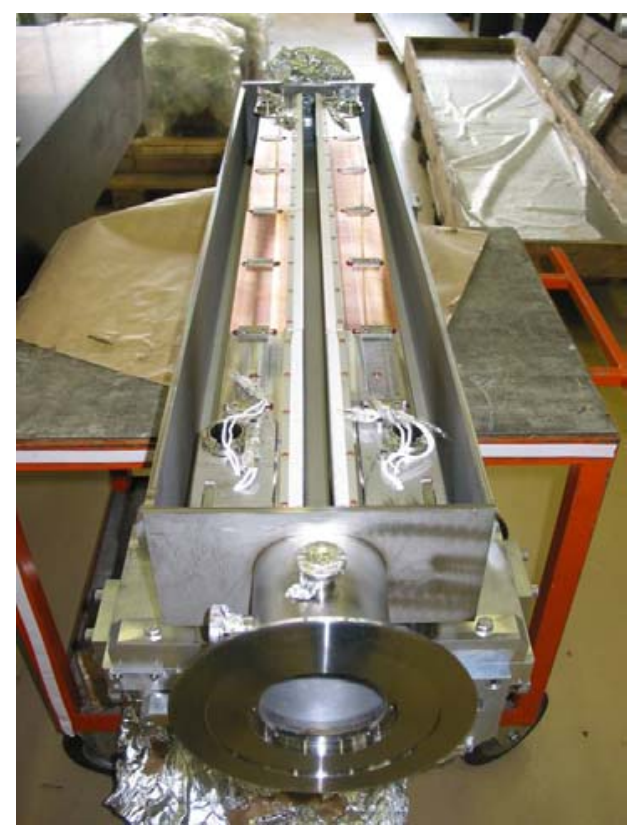

Figure 1: Top view of the open LHC collimator tank with two $1.2 \mathrm{~m}$ long $\mathrm{CC}$ jaws installed.

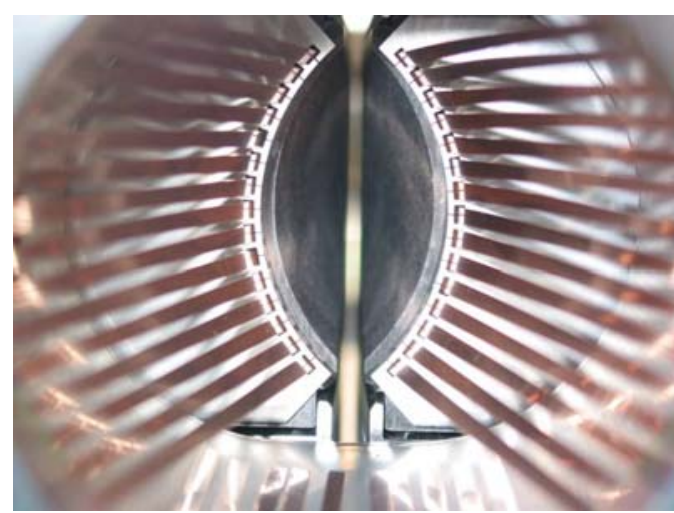

Figure 2: View along the free beam path of the CC collimator with a small collimation gap ( $\sim 2 \mathrm{~mm})$. The RF contacts guide the beam image currents.

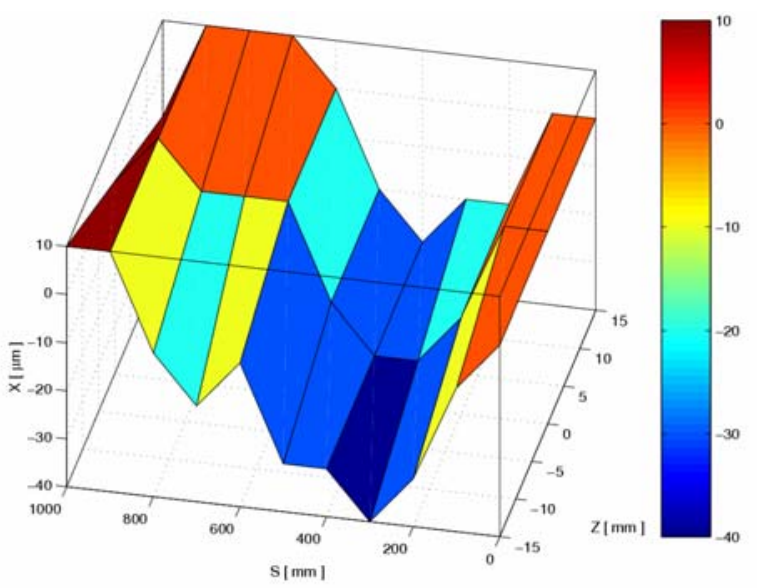

Figure 3: Flatness of the fully assembled CC jaw over its $1 \mathrm{~m}$ long (S) flat top and for three positions along its width (Z). This was measured after bake-out at $250{ }^{\circ} \mathrm{C}$.
Extensive simulations with beam tracking, showering (FLUKA) and thermo-mechanical (ANSYS) codes were relied on for optimizing the collimator design. The prototypes were then extensively measured in the laboratory for evaluating the achieved results. Of particular importance is the flatness of the assembled collimator jaws. A tolerance of $25 \mu \mathrm{m}$ was specified based on the small $7 \mathrm{TeV}$ beam size $\left(\sigma_{\mathrm{x}, \mathrm{y}} \approx 200 \mu \mathrm{m}\right)$ and the $1 \sigma$ difference between primary and secondary collimator positions. A jaw flatness of $40 \mu \mathrm{m}$ was achieved, as shown in Figure 3. Though not as good as specified, the achieved flatness is considered as an excellent success and as acceptable for phase 1 operation in the LHC. Beyond the flatness a number of other important parameters were measured in the laboratory:

1. The electrical resistivity of the $\mathrm{CC}$ jaw was measured to be $10.2 \mu \Omega \mathrm{m}$ along the jaw surface (both parallel and perpendicular to the beam direction). A smaller resistivity of 5-6 $\mu \Omega \mathrm{m}$ was measured in the direction towards the back of the jaw.

2. The thermal conductance through the $\mathrm{CC}$ jaw with the clamped water cooling circuit was tested in a dedicated test stand. For a spring pressure of 6 bar a thermal conductance of $8.8 \mathrm{~kW} / \mathrm{m}^{2} / \mathrm{K}$ was measured.

3. The wear, contact resistance and heating effects for the $0.5 \mathrm{~mm}$ thick $\mathbf{C u B e} \mathbf{R F}$ fingers (coated with $8 \mu \mathrm{m} \mathrm{Ag}$ ) were tested in a dedicated test stand. Contact resistance as low as $0.5 \mathrm{~m} \Omega$ was achieved for the full bridge from $\mathrm{CC}$ to the flanges.

4. The mechanical play in the jaw movement was evaluated for each support point. Plays between $10 \mu \mathrm{m}$ and $40 \mu \mathrm{m}$ were measured with several precise sensors (LVDT, capacitive gauge, resolver).

It was found that the prototype collimators had properties close to the demanding specifications. The design choices were confirmed and the collimators were installed on schedule for beam testing.

\section{RESULTS OF BEAM TESTS}

\section{Robustness test}

A beam line location at the exit from the SPS towards the LHC allows extracting $3 \times 10^{13}$ protons at $450 \mathrm{GeV}$ onto a collimator. The pulse length is $7 \mu \mathrm{s}$ and the transverse size of the extracted beam is $0.7 \times 1.2 \mathrm{~mm}^{2}$. It is interesting to note that the $2 \mathrm{MJ}$ of extracted energy corresponds to the full Tevatron beam or $0.5 \mathrm{~kg}$ of TNT. The LHC collimator was designed to survive twice this energy. Robustness considerations included the $\mathrm{CC}$ jaw, metallic support parts and the water circuit at the $\mathrm{CC}$ jaw. The collimator was repeatedly hit with the $2 \mathrm{MJ}$ beam ( 10 times at highest intensity) with distances up to $5 \mathrm{~mm}$ from the jaw edge. No sign of damage was observed, except the progressing loss of temperature sensors in the CC jaws (see also [3]). A photograph of the collimator after the robustness test is shown in Figure 4. Vibration and sound measurements during the tests are discussed in [8]. The robustness of the collimator design was confirmed. 


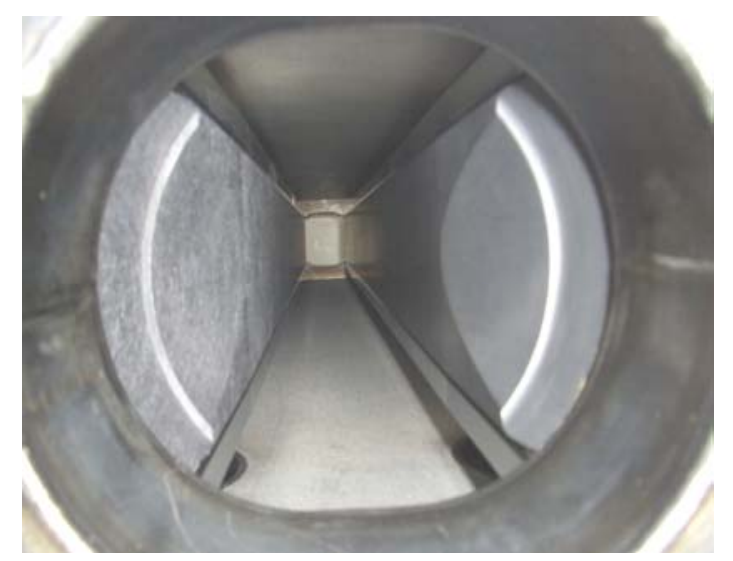

Figure 4: Photograph of the downstream end of the collimator prototype after it was hit repeatedly by a $2 \mathrm{MJ}$ beam, up to $5 \mathrm{~mm}$ from the jaw edge. The left jaw (structured surface) is CC and the right jaw simple Graphite.

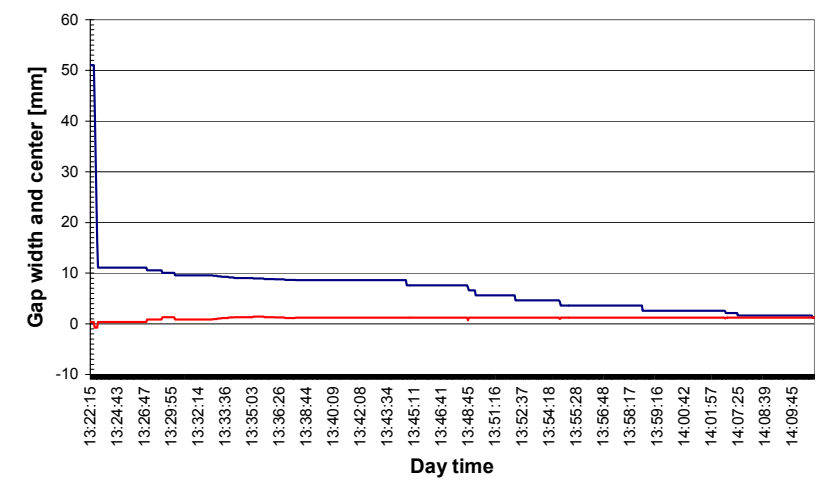

Figure 5: Centre and width of the collimation gap in the SPS ring with stored beam at $270 \mathrm{GeV}$.

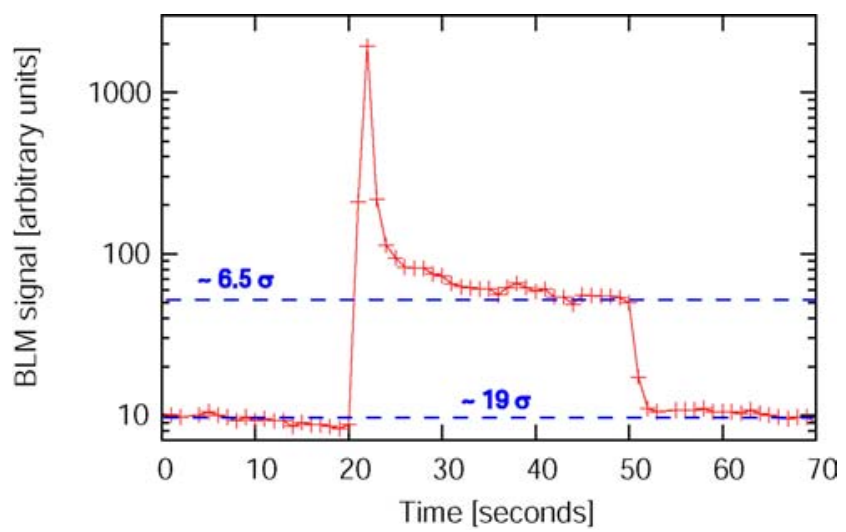

Figure 6: Measured beam loss signal after changing the collimator jaw position from $19 \sigma$ to $6.5 \sigma$ and back.

\section{Operational tests}

The stored SPS beam of $270 \mathrm{GeV}$ protons was used for operational tests. The LHC prototype collimator was complemented by various beam loss monitors downstream. The operational tests included measurements of beam loss patterns, beam-based alignment of collimator gaps and impedance measurements. The impedance measurements are described in [9]. Figure 5 shows the successful beam based alignment of the collimator gap around the stored beam. It is seen that the collimation gap could be adjusted to $1 \mathrm{~mm}$ with circulating beam. This gap is smaller than required for the LHC and illustrates the very satisfactory control and alignment of the LHC prototype collimator. The beam-based alignment was performed with 50-100 $\mu \mathrm{m}$ absolute accuracy. Gaps were known to better than $100 \mu \mathrm{m}$ and the reproducibility of settings was about $20 \mu \mathrm{m}$. The accuracy of the beambased alignment was limited by long decay times in the observed beam loss signals. This was proven to be a real beam dynamics effects and is shown in Figure 6.

\section{CONCLUSIONS}

A powerful collimation system has been designed for the LHC. The phase 1 collimation is robust and efficient but impedance limited. The initial system will be upgraded with phase 2 hybrid collimators, which have been fully integrated into the layout and will be installed several years after the LHC start-up.

The phase 1 collimator design has been worked out in detail. Prototyping and beam tests have proven all main features of the collimator design. In particular a good surface flatness and excellent robustness have been demonstrated. The operational tests with stored beam confirmed the full functionality of the collimator and the expected impedance. Beam-based set-up was limited by beamdynamics effects.

Further work will include the series production and installation of all collimators, the definition of the collimation control system, the preparation of collimator commissioning and the design of phase 2 collimators.

\section{REFERENCES}

[1] R. Assmann et al: "Requirements for the LHC collimation system". EPAC 2002. LHC-Project-Report599.

[2] "The LHC design report, Vol.I Chapter 18". CERN2004-003.

[3] A. Bertarelli et al: "Mechanical Design for Robustness of the LHC Collimators". These proceedings.

[4] E. Metral: "Overview of Impedance and SingleBeam Instability Mechanisms". These proceedings.

[5] V. Vlachoudis et al: "Energy Deposition Studies for the Betatron Cleaning Insertion". These proceedings.

[6] M. Brugger et al: "Calculation of Residual Dose Rates and Intervention Scenarios for the LHC Beam Cleaning Insertions--Constraints and Optimization". These proceedings.

[7] G. Robert-Demolaize et al: "A New Version of SixTrack with Collimation and Aperture Interface". These proceedings.

[8] S. Redaelli et al: "Detecting Impacts of Proton Beams on the LHC Collimators with Vibration Measurements". These proceedings.

[9] H. Burkhardt et al: "Measurements of the LHC Collimator Impedance with Beam in the SPS". These proceedings. 